Research Article

\title{
Seasonal Variation in Patch Test Results with European Baseline Series
}

\author{
Aïcha Brahem (iD, ${ }^{1}$ Haifa Aroui, ${ }^{2}$ Asma Gaddour, ${ }^{2}$ Asma Chouchene, ${ }^{1}$ Asma Aloui, ${ }^{1}$ \\ Imen Kacem, ${ }^{1}$ Maher Maoua, ${ }^{1}$ Houda Kalboussi, ${ }^{1}$ Olfa ElMaalel, ${ }^{1}$ Souhail Chatti, ${ }^{1}$ \\ Faten Dabbabi, ${ }^{1}$ and Nejib Mrizek ${ }^{1}$ \\ ${ }^{1}$ Department of Occupational Medicine, University Hospital Farhat Hached, Faculty of Medicine of Sousse, University of Sousse, \\ Sousse, Tunisia \\ ${ }^{2}$ Depatment of Occupational Medicine, University Hospital Ibnou El Jazzar, Faculty of Medicine of Sousse, University of Sousse, \\ Sousse, Tunisia
}

Correspondence should be addressed to Aïcha Brahem; brahemaicha@gmail.com

Received 1 June 2020; Revised 30 September 2020; Accepted 4 November 2020; Published 16 November 2020

Academic Editor: Markus Stucker

Copyright (C) 2020 Aïcha Brahem et al. This is an open access article distributed under the Creative Commons Attribution License, which permits unrestricted use, distribution, and reproduction in any medium, provided the original work is properly cited.

\begin{abstract}
Aim. To study the influence of season on patch tests results. Methods. We conducted a retrospective epidemiological study which concerned all the patients of the Tunisian center, who consulted in the Dermato-Allergology Unit of Occupational Medicine Department of Farhat Hached University Hospital-Sousse (Tunisia) over a period of 07 years. All the patients were tested by the European Standard Battery allergens (BSE). Results. The data of 1000 patch tests were analyzed during the study period. More than half of the patch tests $(58.6 \%)$ was positive. In winter, $63 \%$ of patch tests showed a positive reaction versus $52 \%$ of patch tests in summer without a statistically significant association. However, results of lanolin alcohols, epoxy resin, and Sesquiterpene lactone mix varied significantly with season. Atopy was significantly associated with $18.8 \%$ of positive reactions in winter and only with $5.2 \%$ of positive reactions in summer $(p=0.015)$. Conclusion. Seasonal variations in patch tests results were more significant with some allergens of European Standard Battery and in atopic patients.
\end{abstract}

\section{Introduction}

Patch test is the gold standard diagnostic procedure for type IV sensitizations; it enables allergen avoidance and promotes secondary prevention of allergic contact dermatitis. However, its reproducibility may be compromised [1].

The relevance of patch tests results depends on several factors: individual (atopy, skin irritation, etc) and external (ultraviolet exposure, drugs, season, etc) [2].

Concerning seasonal variability of patch test results, there is conflicting evidence and no evidence concerning the influence of season on weak positive, possibly false positive, irritant reactions [2].

Previous studies focused on a potential inhibition of patch test reactions caused by ultraviolet exposure [3-7] which is known to protect the skin from contact dermatitis by suppressing the immune reaction and increasing the barrier function of the skin [8].

Other clinical and experimental studies have found increased reactivity to patch tests in relation to winter conditions [9-11]. Low ambient temperature and humidity might diminish epidermal barrier function, leading to overall increased irritability and patch test reactivity [1].

In this context, we carried out an epidemiological study to determine the influence of season on patch tests results with European Standard Battery and to identify seasonal variation of allergens.

\section{Materials and Methods}

We conducted a retrospective epidemiological study which concerned all the patients of the Tunisian center, who 
consulted in the Dermato-Allergology Unit of Occupational Medicine Department of Farhat Hached University Hospital-Sousse (Tunisia) over a period of 07 years. All the patients were tested by the European Standard Battery allergens (BSE).

They were applied on the upper back of patients, using Finn Chambers patches. Test results were coded based on the intensity following the criteria from the International Contact Dermatitis Research Group [12].

Data were collected using a preestablished questionnaire covering socio-demographic and occupational characteristics, past illness history, atopy, and results of the patch-tests.

We analyzed the seasonal variations of the patch-test results within autumn (September-November), winter (December-February), spring (March-May), and summer (June-August).

We also considered seasonality of each allergen reaction: doubtful, weak positive, strong positive, extreme positive, and negative reactions.

Statistical analysis was done using SPSS software. $p$ value threshold was set to 0.05 .

\section{Results}

During the study period, 1006 cases of suspected contact dermatitis (55.7\% women and $44.3 \%$ men) were enrolled. The mean age of our population was $38.9 \pm 13$ years old. About three quarters of the cases ( $76.4 \%$ ) had a job. A history of atopy was noted in $8 \%$ of cases.

Of the 1006 patch tests performed, 58.6\% were positive to at least one allergen; $18.5 \%$ of tests were positive in spring, $11.5 \%$ in summer, $14.8 \%$ in autumn, and $13.8 \%$ in winter.

The allergens with highest frequency of positive reactions were metals in $60 \%$ of cases followed by rubber chemicals in $10.5 \%$ of cases and preservatives in $9.6 \%$ of cases.

In winter, $63 \%$ of patch tests showed a positive reaction versus $52 \%$ of patch tests in summer (Figure 1) without a statistically significant association $(p=0.10)$.

In the cases of some allergens results, significant associations were found. Doubtful reactions to Sesquiterpene lactone mix increased in winter $(p=0.05)$, weak positive reactions to lanolin alcohols increased in spring $(p=0.02)$, and strong positive reactions to epoxy resin increased in summer $(p=0.04)$ (Figure 2).

Atopy was associated with $18.8 \%$ of positive reactions in winter and only $5.2 \%$ of positive reactions in summer with a statistically significant relationship ( $p=0.015)$. On the other hand, neither age nor gender was associated with seasonal reactivity of patch tests.

\section{Discussion}

Our study analyzed 1006 patch tests during seven years. Positive reactions of patch tests with European baseline series increased the most in winter without a statistically significant association. However, some allergens results indicated a significant seasonal variability.

Patch test reactions are relatively stable in the climatic conditions of Tunisia for most allergens. These findings were

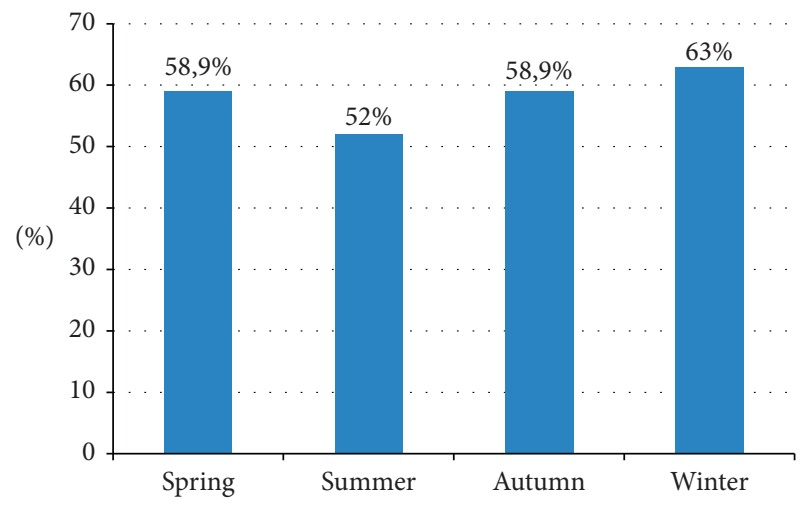

Figure 1: Patch test positive reactions within season.

in agreement with those of Dooms-Goossens et al. [13]. Katsarou et al. [14] explained the no significant influence on patch tests reactions in Athens by small differences in climate conditions that exist between summer and winter in their country.

On another side, a variation of patch test results was reported depending on season. Contact allergens displayed distinctive seasonal patterns $[5,7]$. Similarly, metal patch test reactions were associated with environmental factors, namely, temperature and absolute humidity [8]. Various studies have shown that during winter, the number of positive reactions to allergens is rising, particularly allergens which act simultaneously as mild irritants [15].

Possible explanations for inconsistent weather reaction associations may be the physical properties of the haptens. Differences in how each contact allergen penetrates the skin and produces irritant or allergic reactions could account for their unique relations with weather. The results of previous research indicated that irritant properties may play an important role [8].

Loffler and Happle [15] as well as Agner and Serup [16] investigated a possible seasonal variation in the skin response to irritants, using an irritant patch test with sodium lauryl sulphate (SLS). They showed stronger reactions to SLS during the winter compared to the summer as indicated by measurements of transepidermal water loss. Basketter et al. [17] patch tested sodium dodecyl sulphate (SDS) in 100 volunteers. They reported that the effect of the weather on the intensity of irritant reactivity was evident ( $45 \%$ of the panel reacted to SDS in summer, $91 \%$ reacted in winter). The risk of developing irritant hand dermatitis during the cold months is nearly three times as much as the risk during the warm months as shown by Callahan et al. [18].

The mechanism by which winter conditions (cold and dry air) increased irritant skin changes is identified by previous investigations. These environmental conditions lead to poor epidermal hydration in winter. A low state of hydration can alter the epidermal barrier function and thus predispose it to irritant contact dermatitis [9]. Another study demonstrated that the cutaneous allergic reaction was regulated by environmental humidity and suggested two possible mechanisms of immune regulation: stimulation of Langerhans cells and increased penetration of allergen with low humidity [19]. 

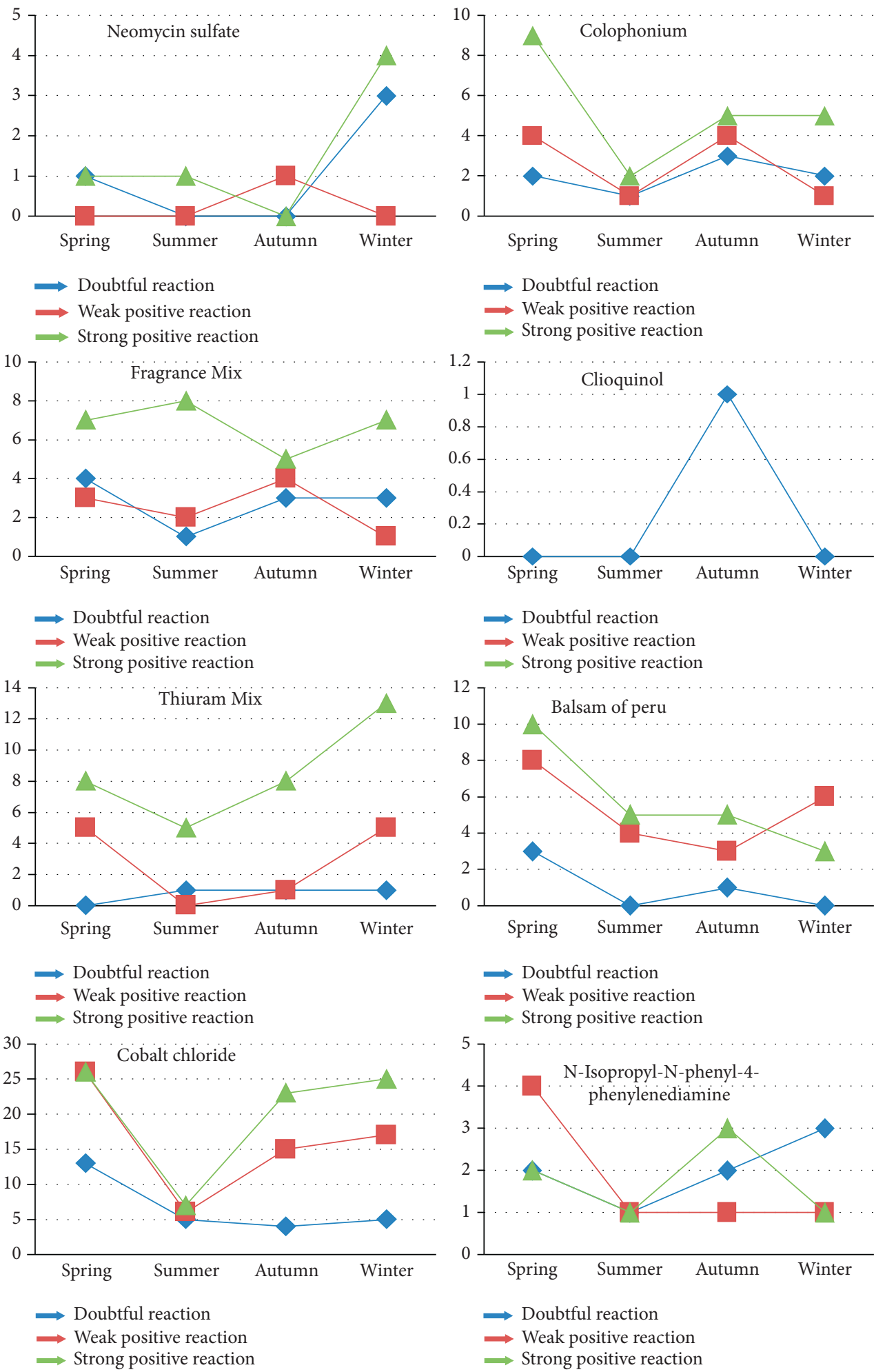

(a)

FIgUre 2: Continued. 

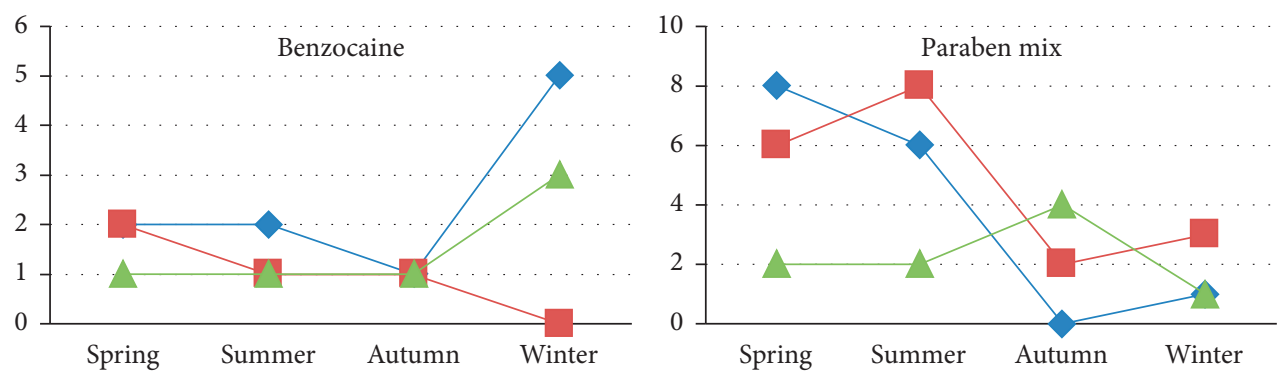

Doubtful reaction

$\longrightarrow$ Weak positive reaction

$\longrightarrow$ Doubtful reaction

$\longrightarrow$ Strong positive reaction

$\longrightarrow$ Weak positive reaction

$\longrightarrow$ Strong positive reaction
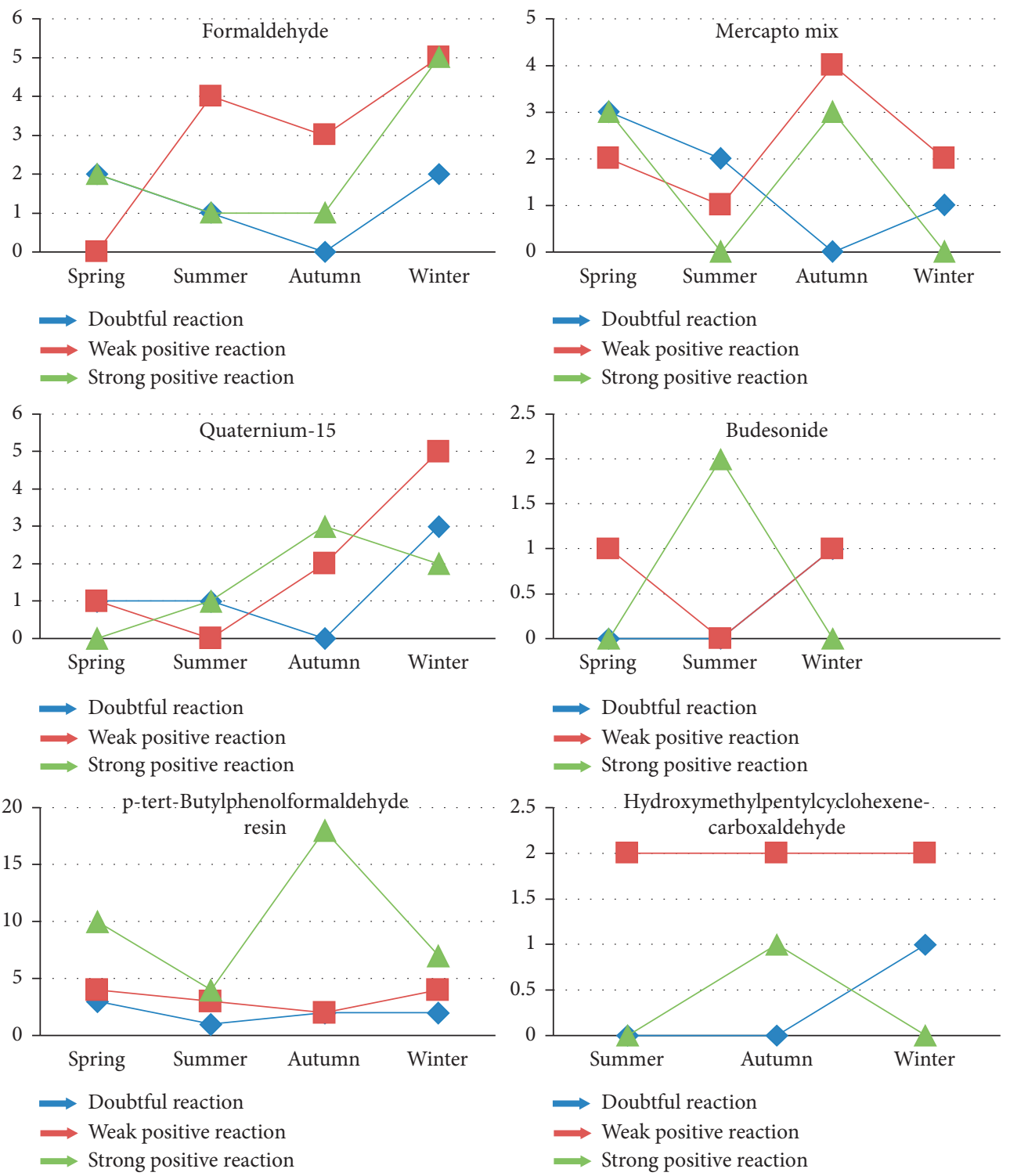

(b)

FIgURE 2: Continued. 

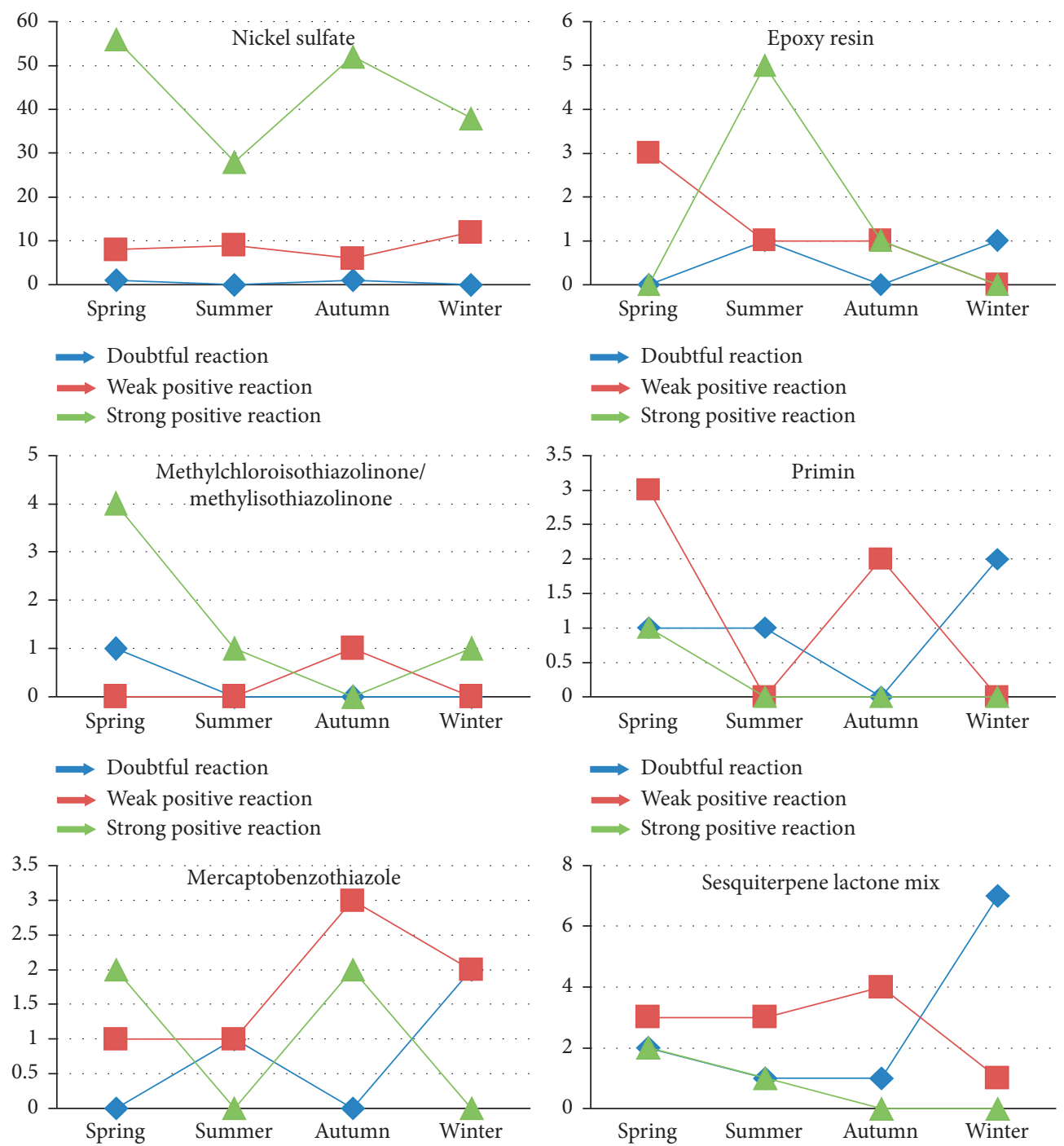

$\longrightarrow$ Doubtful reaction

$\longrightarrow$ Weak positive reaction

$\longrightarrow$ Strong positive reaction

$\longrightarrow$ Doubtful reaction

$\longrightarrow$ Weak positive reaction

$\Longrightarrow$ Strong positive reaction
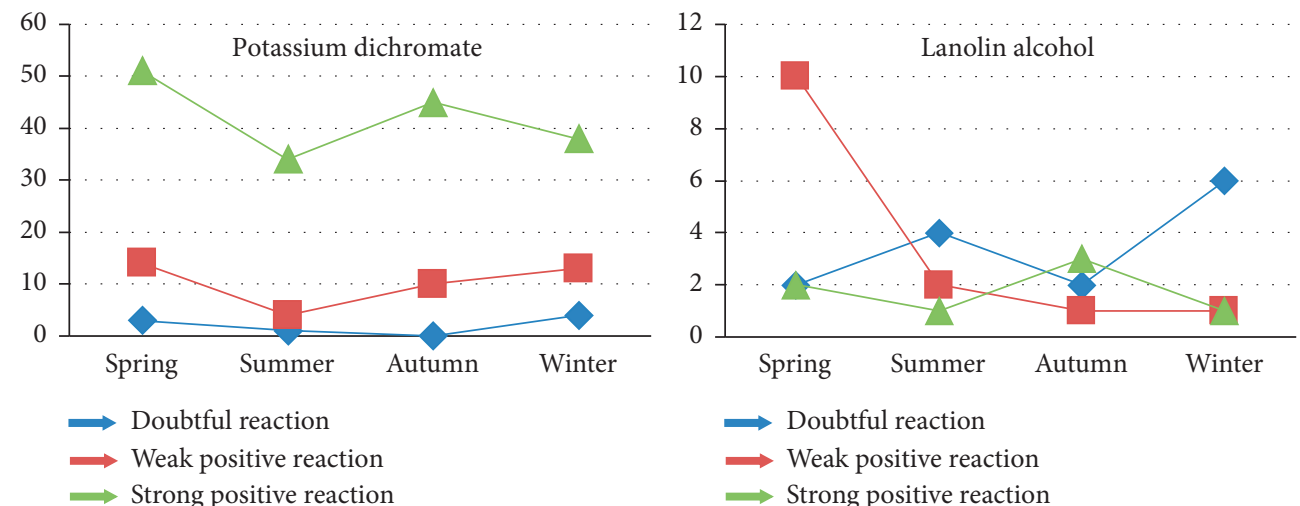

(c)

FIgURE 2: Patch tests results of different allergens according to the season. 
In our study, the influence of seasonal variations on the patch test results was only observed with lanolin alcohols, epoxy resin, and Sesquiterpene lactone mix.

Uter et al. [2] showed that only formaldehyde exhibited a distinct increase in doubtful or irritant as well as weak positive reactions associated with dry cold weather. In a later study published in 2008, the authors noticed a relevant increase of irritant/doubtful reaction frequency in the cases of paraben mix and methylisothiazolinone for low temperature and humidity; the positive reactions $(++/+++)$ of fragrance mix were significantly overrepresented in the two coldest and driest categories [1]. This was explained by the impaired epidermal barrier function under these ambient conditions which renders the skin more vulnerable to the slight irritating effect that many allergens may exert under patch test conditions.

The authors also suggested that water-based allergen preparations were more seasonally dependent than petrolatum-based test materials or, conversely, that petrolatum as patch test vehicle improves the epidermal barrier function in dry/cold ambient conditions [1].

In our study, atopy was significantly associated with positive reactions in winter more than in summer. In Germany, atopic dermatitis was also associated with positive patch tests in about $20 \%$ of cases in winter and in about $17 \%$ of cases in summer [2]. However, according to a previous study, atopic dermatitis was considered as a confounder factor, as consultation peaks in winter [8].

In summer, ultraviolet radiation exposure appears to interfere with the reactivity of patch tests through a suppressive effect on the induction and elicitation of allergic contact dermatitis [6]. As a result, such a situation can lead to false negative tests. It is recommended for patients who have just been subjected to recent or extreme radiation to postpone patch test at a later date [20].

\section{Conclusion}

The results of our study are consistent with those of the literature with a higher frequency of positive reactions of patch tests during the cold season. For most of the allergens, the reactivity of patch test was relatively stable in Tunisia's temperate climate. However, some allergens were affected by seasonal variations. The increase in doubtful and weakly positive reactions for several allergens could be explained by the impairment of the epidermal barrier function under winter climatic conditions. In that case, relevance of these doubtful/irritant reactions is uncertain during winter, especially in patients with a history of atopy. They should be retested under warm conditions.

\section{Data Availability}

The data are available upon request to the corresponding authors.

\section{Conflicts of Interest}

The authors declare that they have no conflicts of interest.

\section{References}

[1] W. Uter, J. Hegewald, B. Kränke, A. Schnuch, O. Gefeller, and A. Pfahlberg, "The impact of meteorological conditions on patch test results with 12 standard series allergens (fragrances, biocides, topical ingredients)," British Journal of Dermatology, vol. 158, no. 4, pp. 734-739, 2008.

[2] W. Uter, J. Geier, M. Land, A. Pfahlberg, O. Gefeller, and A. Schnuch, "Another look at seasonal variation in patch test results," Contact Dermatitis, vol. 44, no. 3, pp. 146-152, 2001.

[3] M. Bruze, "Seasonal influence on routine patch test results," Contact Dermatitis, vol. 14, no. 3, p. 184, 1986.

[4] B. Edman, "Systemic drugs and palmar eczema," Current Topics in Contact Dermatitis, vol. 20, p. 226, 1989.

[5] N. Hjorth, "Seasonal variations in contact dermatitis," Acta Dermato-Venereologica, vol. 47, pp. 407-418, 1967.

[6] A. Ingber, A. Sasson, and M. David, "The seasonal influence on patch test reactions is significant in Israel," Contact Dermatitis, vol. 39, no. 6, pp. 318-319, 1998.

[7] B. Kränke and W. Aberer, "Seasonal influence on patch test results in central Europe," Contact Dermatitis, vol. 34, no. 3, pp. 215-231, 1996.

[8] J. Hegewald, W. Uter, B. Kränke, A. Schnuch, O. Gefeller, and A. Pfahlberg, "Patch test results with metals and meteorological conditions," International Archives of Allergy and Immunology, vol. 147, no. 3, pp. 235-240, 2008.

[9] W. Uter, O. Gefeller, and H. J. Schwanitz, "An epidemiological study of the influence of season (cold and dry air) on the occurrence of irritant skin changes of the hands," British Journal of Dermatology, vol. 138, no. 2, pp. 266-272, 1998.

[10] S. M. John and W. Uter, "Meteorological influence on $\mathrm{NaOH}$ irritation varies with body site," Archives of Dermatological Research, vol. 296, no. 7, pp. 320-326, 2005.

[11] H. Löffler, C. Pirker, and J. Aramaki, "Evaluation of skin susceptibility to irritancy by routine patch testing with sodium lauryl sulfate," European Journal of Dermatology, vol. 11, pp. 416-419, 2001

[12] D. S. Wilkinson, S. Fregert, B. Magnusson et al., "Terminology of contact dermatitis," Acta Dermato-Venereologica, vol. 50, pp. 287-292, 1970.

[13] A. Dooms-Goossens, E. Lesaffre, M. Heidbuchel, M. Dooms, and H. Degreef, "UV sunlight and patch test reactions in humans," Contact Dermatitis, vol. 19, no. 1, pp. 36-42, 1988.

[14] A. Katsarou, V. Koufou, D. Kalogeromitros, M. Armenaka, D. Papaioannou, and J. Stratigos, "Seasonal influence on patch test results in Greece," Photodermatol Photoimmunol Photomed, vol. 9, pp. 232-234, 1993.

[15] H. LÖffler and R. Happle, "Influence of climatic conditions on the irritant patch test with sodium lauryl sulphate," Acta Dermato-Venereologica, vol. 83, no. 5, pp. 338-341, 2003.

[16] T. Agner and J. Serup, "Seasonal variation of skin resistance to irritants," British Journal of Dermatology, vol. 121, no. 3, pp. 323-328, 1989.

[17] D. A. Basketter, H. A. Griffiths, X. M. Wang, K.-P. Wilhelm, and J. McFadden, "Individual, ethnic and seasonal variability in irritant susceptibility of skin: the implications for a predictive human patch test," Contact Dermatitis, vol. 35, no. 4, pp. 208-213, 1996.

[18] A. Callahan, E. Baron, D. Fekedulegn et al., "Winter season, frequent hand washing, and irritant patch test reactions to detergents are associated with hand dermatitis in health care workers," Dermatitis, vol. 24, no. 4, pp. 170-175, 2013. 
[19] J. Hosoi, T. Hariya, M. Denda, and T. Tsuchiya, "Regulation of the cutaneous allergic reaction by humidity," Contact Dermatitis, vol. 42, no. 2, pp. 81-84, 2000.

[20] J.-M. Lachapelle, "Patch-tests dans différentes conditions climatiques," Annales de Dermatologie et de Vénéréologie, vol. 136, no. 8-9, pp. 621-622, 2009. 